\begin{tabular}{|c|c|}
\hline $\begin{array}{l}\text { 3. From: coriginating organization) } \\
\text { TWRS Projects/SST Retrieval }\end{array}$ & $\begin{array}{r}\text { 4. Related EDr No.: } \\
\text { n/a }\end{array}$ \\
\hline $\begin{array}{l}\text { 6. Design Authority/ Design Agent/Cog. } \\
\text { Engr:: } \\
\text { JW Bailey, NHC }\end{array}$ & $\begin{array}{l}\text { 7. Purchase Order No.: } \\
\qquad \mathrm{n} / \mathrm{a} \\
\end{array}$ \\
\hline \multirow{2}{*}{$\begin{array}{l}\text { 8. Originator Remarks: } \\
\text { For approval and release of a new supporting document. This } \\
\text { document has been generated to ensure retrievability of the } \\
\text { Project } W-320 \text { "Master Calculation List". }\end{array}$} & $\begin{array}{l}\text { 9. Equip./Component No.: } \\
n / \mathrm{a}\end{array}$ \\
\hline & $\begin{array}{c}\text { 10. System/8ldg./Facility: } \\
241-C-106 \\
\end{array}$ \\
\hline \multirow[t]{3}{*}{ 11. Receiver Remarks: 11A. Design Baseline Document? [] Yes $[X]$ No } & $\begin{array}{l}\text { 12. Major Assm. Dwg. No.: } \\
n / a\end{array}$ \\
\hline & $\begin{array}{l}\text { 13. Permit/Permit Application No.: } \\
n / a\end{array}$ \\
\hline & 14. Required Response Date: \\
\hline
\end{tabular}

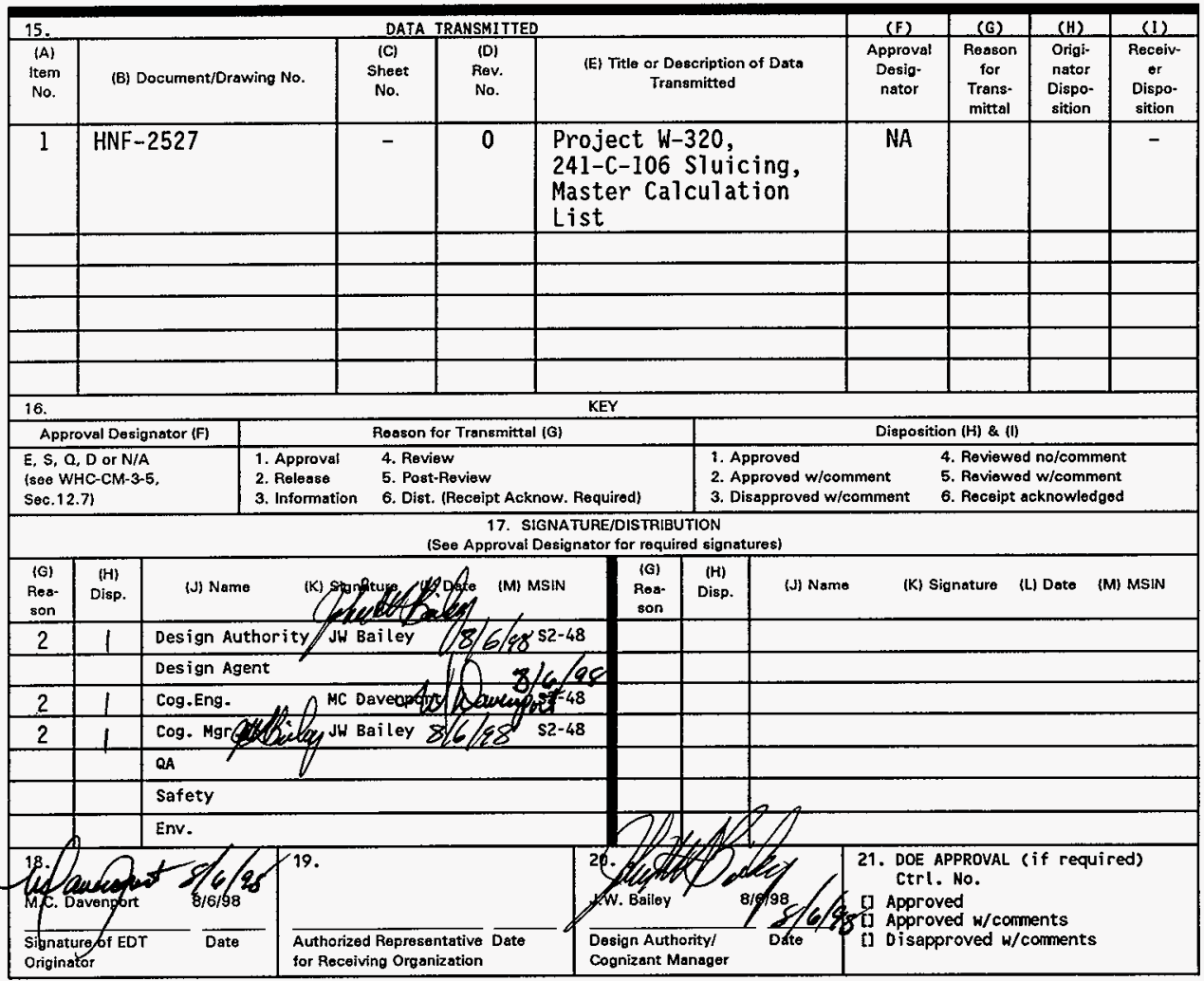




\section{Project W-320, 241-C-106 Sluicing, Master Calculation List}

John W. Bailey

Numatec Hanford Co., Richland, WA 99352

U.S. Department of Energy Contract DE-AC09-96RL13200
EDT/ECN: 624730
UC: 506
Org Code: $8 \mathrm{C} 452$
Charge Code: D2991/HANA0600
B\&R Code: EW3130010
Total Pages: $/ 8$

Key Words: W-320, Sluicing, Tank 241-C-106, Tank 241-AY-102, Calculation, WRSS.

Abstract: This supporting document has been prepared to make the "Master Calculation List" readily retrievable.

TRADEMARK DISCLAIMER. Reference herein to any specific commercial product, process, or service by trade name, trademark, manufacturer, or otherwise, does not necessarily constitute or imply its endorsement, recomendation, or favoring by the United States Government or any agency thereof or its contractors or subcontractors.

Printed in the United States of America. To obtain copies of this document, contact: Document Control Services, P.0. Box 950, Mailstop H6-08, Richland WA 99352, Phone (509) 372-2420; Fax (509) 376-4989.
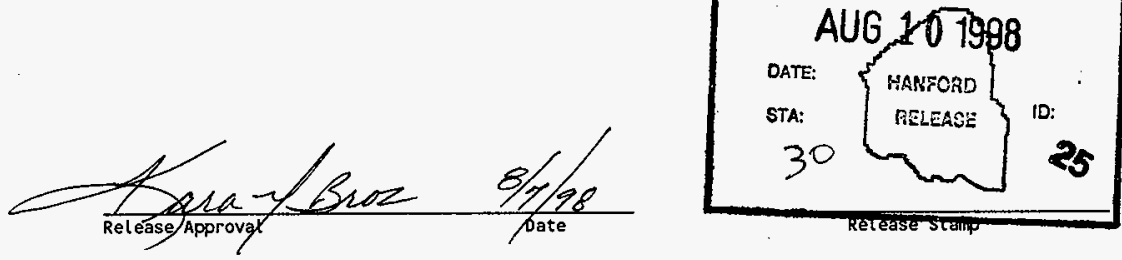
Project W-320 Master Calculation List

\begin{tabular}{|c|c|c|c|c|c|c|c|c|}
\hline & $\begin{array}{l}\text { Calc No. } \\
\text { W-320- }\end{array}$ & Rev & Status & Discpl & Calculation Title & Originator & Comments & $\begin{array}{l}\text { Issued } \\
\text { Under } \\
\text { HNF. }\end{array}$ \\
\hline 1 & $21-001$ & 0 & As-Built & $\begin{array}{l}\text { Civ } / \\
\text { Struct }\end{array}$ & Drainage Run-Off - Size Culverts & PC Brinkman & Site work: & 2461 \\
\hline 2 & $21-002$ & 0 & App'd & $\begin{array}{l}\text { Civ/ } \\
\text { Struct }\end{array}$ & Site Drainage- Interfarm \& C-Farm & MR Custer & Site work: & 2461 \\
\hline 3 & 24-001 & 1 & As-Built & $\begin{array}{l}\text { Civ } / \\
\text { Struct }\end{array}$ & Cold Test Facility Mock-ups & JG Rupert & $\begin{array}{l}\text { Equipment Removal Sys: W-106 Caisson, } \\
\text { retained for record only and not listed on the } \\
\text { drawing index }\end{array}$ & 2461 \\
\hline 4 & 24-002 & 1 & As-Built & $\begin{array}{c}\text { Civ/ } \\
\text { Struct }\end{array}$ & $\begin{array}{l}\text { Flex. Receiver Suppt \& Shielded Work } \\
\text { Platform - sluicing Pit 241-AY-02B }\end{array}$ & & $\begin{array}{l}\text { Equipment Removal Sys: Pit 241-AY-O2B, } \\
\text { retained for record only and not listed on the } \\
\text { drawing index }\end{array}$ & 2462 \\
\hline 5 & 24-003 & 1 & As-Built & $\begin{array}{l}\text { Civ/ } \\
\text { Struct }\end{array}$ & $\begin{array}{l}\text { Flexible Receiver Suppt \& Shielded } \\
\text { Work Platform - Pump Pit 241-CR-06A }\end{array}$ & MB Lasota & $\begin{array}{l}\text { Equipment Removal Sys: Pit 241-CR-06A, } \\
\text { retained for record only and not listed on the } \\
\text { drawing index }\end{array}$ & 2463 \\
\hline 6 & 24-004 & 1 & As-Built & $\begin{array}{l}\text { Civ/ } \\
\text { Struct }\end{array}$ & $\begin{array}{l}\text { Flexible Receiver Suppt \& Shielded } \\
\text { Work Platform - Heel Pit 241-CR-06B }\end{array}$ & MB Lasota & Equipment Removal Sys: Pit 241-CR-06B & 2464 \\
\hline 7 & $24-005$ & 0 & As-Built & $\begin{array}{l}\text { Civ/ } \\
\text { Struct }\end{array}$ & $\begin{array}{l}\text { Design Two Electrical Equipment Skids } \\
\text { and One Concrete Slab }\end{array}$ & JG Rupert & $\begin{array}{l}\text { Equipment Removal Sys: Transfer } \\
\text { Switch/Breakers Skid; XFMR/Breaker Skid }\end{array}$ & 2461 \\
\hline 8 & 24-006 & 0 & As-Built & $\begin{array}{c}\text { Civ/ } \\
\text { Struct }\end{array}$ & Distribution Panel Skid \& Pad Design & JR Booth & $\begin{array}{l}\text { Equipment Removal Sys: Distribution Panel } \\
\text { Skid }\end{array}$ & 2461 \\
\hline 9 & $24-007$ & 0 & As-Built & $\begin{array}{l}\text { Civ/ } \\
\text { Struct }\end{array}$ & $\begin{array}{l}\text { Design Bracket that Clamps to a Crane } \\
\text { Boom to Support a Camera Pan/Tilt }\end{array}$ & JG Rupert & $\begin{array}{l}\text { Equipment Removal Sys: Camera Pan Tilt, } \\
\text { retained for record only and not listed on the } \\
\text { drawing index }\end{array}$ & 2461 \\
\hline 10 & 24-008 & & $\begin{array}{l}\text { Not } \\
\text { Used }\end{array}$ & & Not Used & & Not Used & NA \\
\hline 11 & 24-009 & & $\begin{array}{c}\text { Not } \\
\text { Used }\end{array}$ & & Not Used & & Not Used & NA \\
\hline 12 & $24-010$ & & $\begin{array}{l}\text { Not } \\
\text { Used }\end{array}$ & & Not Used & & Not Used & NA \\
\hline 13 & 24-011 & 1 & As-Built & $\begin{array}{l}\text { Civ } / \\
\text { Struct }\end{array}$ & $\begin{array}{l}\text { Pump Pit C-06A Analysis and Cover } \\
\text { Block Design }\end{array}$ & MB Lasota & C-Farm: Pit C-06A; ECN W-320-192 & 2465 \\
\hline 14 & 24-012 & & $\begin{array}{l}\text { Not } \\
\text { Used }\end{array}$ & & Not Used & & Not Used & NA \\
\hline 15 & 24-013 & 0 & App'd & $\begin{array}{c}\text { Civ/ } \\
\text { Struct }\end{array}$ & $\begin{array}{l}\text { Sluice Pit C-06C Analysis of The Pit } \\
\text { Wall }\end{array}$ & MB Lasota & C-Farm: Pit C-06C; See Calc W-320-24-011 & 2465 \\
\hline 16 & 24-014 & 1 & As-Built & $\begin{array}{l}\text { Civ/ } \\
\text { Struct }\end{array}$ & $\begin{array}{l}\text { Process Building \& Equipment Skid } \\
\text { Foundation }\end{array}$ & MR Custer & $\begin{array}{l}\text { C-Farm: 241-C-91; Non-Complex SC3 } \\
\text { Structure }\end{array}$ & 2465 \\
\hline
\end{tabular}


Project W-320 Master Calculation List

\begin{tabular}{|c|c|c|c|c|c|c|c|c|}
\hline & $\begin{array}{l}\text { Calc No. } \\
\text { W-320- }\end{array}$ & Rev & Status & Discpl & Calculation Title & Originator & Comments & $\begin{array}{l}\text { Issued } \\
\text { Under } \\
\text { HNF. }\end{array}$ \\
\hline 17 & 24-015 & 1 & App'd & $\begin{array}{l}\text { Civ } / \\
\text { Struct }\end{array}$ & 241-C-106 Dome Load Summary Report & RW Davidson & C-Farm: Tank 241-C-106 & 2466 \\
\hline 18 & 24-016 & 2 & As-Built & $\begin{array}{c}\text { Civ/ } \\
\text { Struct }\end{array}$ & Sluice Pit AY-02E Analysis & MB Lasota & $\begin{array}{l}\text { AY-Farm: Pit AY-02E; Design Verified (Simple } \\
\text { SC 1) }\end{array}$ & 2466 \\
\hline 19 & 24-017 & 2 & App'd & $\begin{array}{c}\text { Civ/ } \\
\text { Struct }\end{array}$ & $\begin{array}{l}\text { Pump Pit AY-02A Analysis/New Cover } \\
\text { Design }\end{array}$ & MB Lasota & AY-Farm: Pit AY-02A; ECN W-320-319 & 2466 \\
\hline 20 & $24-018$ & 1 & As-Built & $\begin{array}{l}\text { Civ/ } \\
\text { Struct }\end{array}$ & $\begin{array}{l}\text { 241-AY-102 Dome Load Summary } \\
\text { Report }\end{array}$ & RW Davidson & AY-Farm: Tank 241-AY-102 & 2466 \\
\hline 21 & $24-019$ & 1 & As-Built & $\begin{array}{c}\text { Civ/ } \\
\text { Struct }\end{array}$ & $\begin{array}{l}\text { Prefiltration/Recirculation Skid, } \\
\text { Structural Design (Process Control Bldg) }\end{array}$ & MR Custer & HVAC Skid: 241-C-91; ECN Incorp & 2467 \\
\hline 22 & 24-020 & 1 & As-Built & $\begin{array}{c}\text { Civ/ } \\
\text { Struct }\end{array}$ & $\begin{array}{l}\text { Intake Air Filter Pad \& Cooling Coil } \\
\text { Frame }\end{array}$ & RW Davidson & $\begin{array}{l}\text { C-Farm: Intake Air Filter; SC } 3 \text { Equip. } \\
\text { Pad/Anchorage }\end{array}$ & 2467 \\
\hline 23 & $24-021$ & 2 & As-Built & $\begin{array}{c}\text { Civ/ } \\
\text { Struct }\end{array}$ & $\begin{array}{l}\text { Equipment Foundation - Exhaust Air } \\
\text { Cleanup Train }\end{array}$ & RW Davidson & $\begin{array}{l}\text { C-Farm: Exhaust Air Cleanup Train; } \\
\text { SC } 3 \text { Equip. Pad/Anchorage }\end{array}$ & 2467 \\
\hline 24 & $24-022$ & 1 & As-Built & $\begin{array}{c}\text { Civ/ } \\
\text { Struct }\end{array}$ & $\begin{array}{l}\text { Service Building Foundation } \\
\text { (A\&W Bldg.) }\end{array}$ & RW Davidson & $\begin{array}{l}\text { Site work: 241-C-73; Non-Complex SC } 3 \\
\text { Structure }\end{array}$ & 2467 \\
\hline 25 & $24-023$ & 0 & As-Built & $\begin{array}{c}\text { Civ/ } \\
\text { Struct }\end{array}$ & $\begin{array}{l}\text { Equipment Foundation - Chiller and } \\
\text { Pumps }\end{array}$ & MR Custer & $\begin{array}{l}\text { C-Farm: Chiller and Pumps; SC } 3 \text { Equip. } \\
\text { Pad/Anchorage }\end{array}$ & 2467 \\
\hline 26 & $24-024$ & 1 & As-Built & $\begin{array}{c}\text { Civ/ } \\
\text { Struct }\end{array}$ & Electrical Equipment Pad (241-C-51) & RW Davidson & $\begin{array}{l}\text { C-Farm: 241-C-51; SC } 3 \text { Equip. } \\
\text { Pad/Anchorage }\end{array}$ & 2467 \\
\hline 27 & $24-025$ & 1 & App'd & $\begin{array}{c}\text { Civ/ } \\
\text { Struct }\end{array}$ & Cover Block Lifting Bail Evaluation & MB Lasota & C-Farm: & 2468 \\
\hline 28 & $24-026$ & 0 & As-Built & $\begin{array}{c}\text { Civ/ } \\
\text { Struct }\end{array}$ & $\begin{array}{l}\text { Seismic Shutdown System Pad } / \text { Wind } \\
\text { Missile Evaluation }\end{array}$ & JR Booth & $\begin{array}{l}\text { AY- \& C-Farm: 241-AY-51A/51B, 241-C- } \\
51 \mathrm{~A} / 51 \mathrm{~B} \text {; Design Verified (simple SC 3/1) }\end{array}$ & 2468 \\
\hline 29 & $24-027$ & 3 & As-Built & $\begin{array}{c}\text { Civ/ } \\
\text { Struct }\end{array}$ & $\begin{array}{l}\text { Seismic Analysis for Misc. Safety } \\
\text { Related Equipment Attachments }\end{array}$ & MB Lasota & C-Farm: SC 3 Equip. Anchorage & 2468 \\
\hline 30 & $24-028$ & 1 & App'd & $\begin{array}{c}\text { Civ/ } \\
\text { Struct }\end{array}$ & $\begin{array}{l}\text { Engineering Evaluation of Commercial } \\
\text { Grade Items }\end{array}$ & RW Davidson & C-Farm: & 2468 \\
\hline 31 & $24-029$ & 2 & As-Built & $\begin{array}{c}\text { Civ/ } \\
\text { Struct }\end{array}$ & $\begin{array}{l}\text { Structural Analysis of safety Related } \\
\text { Electrical Equipment Supports. }\end{array}$ & MB Lasota & AY- \& C-Farm & 2468 \\
\hline 32 & $24-030$ & 0 & As-Built & $\begin{array}{c}\text { Civ/ } \\
\text { Struct }\end{array}$ & $\begin{array}{l}\text { Electrical Equipment and Conduit } \\
\text { Supports-Process Bldg. Skid }\end{array}$ & MR Custer & HVAC Skid: & 2468 \\
\hline 33 & $24-031$ & 0 & As-Built & $\begin{array}{l}\text { Civ/ } \\
\text { Struct }\end{array}$ & Process Bldg Equip Skid Rigging Frame & MR Custer & $\begin{array}{l}\text { HVAC Skid: } 241-C-91 \text {, retained for record } \\
\text { only and not listed on the drawing index }\end{array}$ & 2468 \\
\hline 34 & 24-032 & 0 & As-Built & $\begin{array}{c}\text { Civ/ } \\
\text { Struct }\end{array}$ & Cover Block Analysis-Sluice Pit AY-02E & MB Lasota & AY-Farm: Pit AY-O2E; ECN W-320-173 & 2468 \\
\hline
\end{tabular}

Page 2 of 17 
Project W-320 Master Calculation List

\begin{tabular}{|c|c|c|c|c|c|c|c|c|}
\hline & $\begin{array}{l}\text { Calc No. } \\
\text { W-320- }\end{array}$ & Rev & Status & Discpl & Calculation Title & Originator & Comments & $\begin{array}{l}\text { Issued } \\
\text { Under } \\
\text { HNF. }\end{array}$ \\
\hline 35 & $24-033$ & 0 & As-Built & $\begin{array}{l}\text { Civ } / \\
\text { Struct }\end{array}$ & Vehicle Load Over Pipeline & RL Ackerman & C-Farm: & 2468 \\
\hline 36 & $24-034$ & 0 & As-Built & $\begin{array}{l}\text { Civ/ } \\
\text { Struct }\end{array}$ & Process Bldg Lower Lifting Frame & MR Custer & $\begin{array}{l}\text { C-Farm: } 241-C-91 \text { retained for record only } \\
\text { and listed on the drawing index }\end{array}$ & 2468 \\
\hline 37 & $24-035$ & 0 & As+Built & $\begin{array}{c}\text { Civ } / \\
\text { Struct }\end{array}$ & $\begin{array}{l}\text { Recirculation/Exhaust Skid Strt } \\
\text { Evaluations }\end{array}$ & NG Ha & C-Farm: Exhaust Air Cleanup Train & 2468 \\
\hline 38 & $24-036$ & 0 & App'd & $\begin{array}{c}\text { Civ/ } \\
\text { Struct }\end{array}$ & Exhaust Skid Platform Modification & AS Langevin & C-Farm: & 2468 \\
\hline 39 & $24-037$ & 0 & App'd & $\begin{array}{c}\text { Civ } / \\
\text { Struct }\end{array}$ & $\begin{array}{l}\text { Check Calcs for AY-102 Electrical } \\
\text { Equipment Rack }\end{array}$ & LA Valle & AY-Farm; & 2468 \\
\hline 40 & $24-038$ & 0 & As-Built & $\begin{array}{c}\text { Civ } 1 \\
\text { Struct }\end{array}$ & $\begin{array}{l}\text { DBE Analysis for } 1000 \text { CFM Portable } \\
\text { Exhauster }\end{array}$ & CA Fargo & $\begin{array}{l}\text { AY- \& C-Farm: Exhausters VTP-POR-007 \& - } \\
008\end{array}$ & 2468 \\
\hline 41 & $25-001$ & 0 & As-Built & Mech & Decontamination Spray Ring Pipe Stress & RC Campbell & $\begin{array}{l}\text { Equipment Removal Sys: Use of Spray Ring } \\
\text { deleted from project. Calc checked against } \\
\text { released drawings, retained for record only } \\
\text { and not listed on the drawing index }\end{array}$ & No \\
\hline 42 & $25-002$ & 1 & As-Built & Mech & $\begin{array}{l}\text { Lifting Fixture Stress Analysis, Transfer } \\
\text { Pump Lifting Fixture }\end{array}$ & RC Campbell & $\begin{array}{l}\text { Equipment Removal Sys: Removal of the } \\
\text { existing Transfer Pump and the use of the } \\
\text { Pump Lif ting Fixture deleted from project. } \\
\text { Calc checked against released drawings, } \\
\text { retained for record only and not listed on the } \\
\text { drawing index }\end{array}$ & No \\
\hline 43 & $25-003$ & 1 & As-Built & Mech & $\begin{array}{l}\text { Lifting Fixture Stress Analysis, Heel Jet } \\
\text { Lif́ting Fixture }\end{array}$ & RC Campbell & $\begin{array}{l}\text { Equipment Remova! Sys: Removal of the } \\
\text { existing Heel Jet and the use of the Heel Jet } \\
\text { Lifting Fixture deleted from project. Calc } \\
\text { checked against released drawings, retained } \\
\text { for record only and not listed on the drawing } \\
\text { index }\end{array}$ & No \\
\hline 44 & $25-004$ & 0 & App'd & Mech & $\begin{array}{l}\text { Center of Gravity, Heel Jet, Tank 106C, } \\
\text { Pit B }\end{array}$ & RC Campbell & $\begin{array}{l}\text { Equipment Removal Sys: Removal of the Heel } \\
\text { Jet deleted from project. Calc given final } \\
\text { review, retained for record only and not listed } \\
\text { on the drawing index }\end{array}$ & No \\
\hline 45 & $25-005$ & 0 & App'd & Mech & $\begin{array}{l}\text { Center of Gravity, Agitator Pump, Tank } \\
\text { 102AY, Pit B }\end{array}$ & RC Campbell & $\begin{array}{l}\text { Equipment Removal Sys: Removal of the } \\
\text { Agitator Pump deleted from project. Calc } \\
\text { given final review, retained for record only } \\
\text { and not listed on the drawing index }\end{array}$ & No \\
\hline
\end{tabular}


Project W-320 Master Calculation List

\begin{tabular}{|c|c|c|c|c|c|c|c|c|}
\hline & $\begin{array}{l}\text { Calc No. } \\
\text { W-320- }\end{array}$ & Rev & Status & Discpl & Calculation Title & Originator & Comments & $\begin{array}{l}\text { Issued } \\
\text { Under } \\
\text { HNF. }\end{array}$ \\
\hline 46 & $25-006$ & 0 & App'd & Mech & $\begin{array}{l}\text { Center Of Gravity, Transfer pump, Tank } \\
\text { 106C, Pit A "Mystery Pump" }\end{array}$ & RC Campbell & $\begin{array}{l}\text { Equipment Removal Sys: Removal ot the } \\
\text { existing Transfer Pump deleted from project. } \\
\text { Calc given final review, retained for record } \\
\text { only and not listed on the drawing index }\end{array}$ & No \\
\hline 47 & $25-007$ & 1 & As-Built & Mech & $\begin{array}{l}\text { Lifting Fixture Stress Analysis, Agitator } \\
\text { Pump Lifting Fixture }\end{array}$ & RC Campbell & $\begin{array}{l}\text { Equipment Removal Sys: Removal of the } \\
\text { existing Agitator Pump and the use of the } \\
\text { Pump Lifting Fixture deleted from project. } \\
\text { Calc checked against released drawings, } \\
\text { retained for record only and not listed on the } \\
\text { drawing index }\end{array}$ & No \\
\hline 48 & $25-008$ & 1 & As-Built & Mech & $\begin{array}{l}\text { Spool Piece Weight, Pump Pit 102AY, } \\
\text { Pit B }\end{array}$ & RC Campbell & $\begin{array}{l}\text { Equipment Removal Sys: Use of the Pit B } \\
\text { spool piece deleted from project. Calc } \\
\text { checked against released drawings, retained } \\
\text { for record only and not listed on the drawing } \\
\text { index }\end{array}$ & No \\
\hline 49 & $25-009$ & 0 & As-Built & Mech & $\begin{array}{l}\text { Spool Piece Weight, Pump Pit 106C, Pit } \\
\text { A }\end{array}$ & RC Campbell & $\begin{array}{l}\text { Equipment Removal Sys: Use of the Pit A } \\
\text { spool piece deleted from project. Calc } \\
\text { checked against released drawings, retained } \\
\text { for record only and not listed on the drawing } \\
\text { index }\end{array}$ & No \\
\hline 50 & $25-010$ & 1 & As-Built & Mech & $\begin{array}{l}\text { Spool Piece Weight, Pump Pit 106C, Pit } \\
\text { B }\end{array}$ & RC Campbell & $\begin{array}{l}\text { Equipment Removal Sys: Use of the Pit B } \\
\text { spool piece deleted from project. Calc } \\
\text { checked against released drawings, retained } \\
\text { for record only and not listed on the drawing } \\
\text { index }\end{array}$ & No \\
\hline 51 & $25-011$ & 0 & As-Built & Mech & Tie Rod Sizing for Spool Pieces & RC Campbelt & $\begin{array}{l}\text { Equipment Removal Sys: Use of spool pieces } \\
\text { deleted from project. Calc checked against } \\
\text { released drawings, retained for record only } \\
\text { and not listed on the drawing index }\end{array}$ & No \\
\hline 52 & 25-012 & 0 & As-Built & Mech & Pump Lifting Point Strength Analysis & RC Campbell & $\begin{array}{l}\text { Equipment Removal Sys: Removal of the } \\
\text { existing Transfer Pump deleted from project. } \\
\text { Calc checked against released drawings, } \\
\text { retained for record only and not listed on the } \\
\text { drawing index }\end{array}$ & No \\
\hline
\end{tabular}


Project W-320 Master Calculation List

\begin{tabular}{|c|c|c|c|c|c|c|c|c|}
\hline & $\begin{array}{l}\text { Calc No. } \\
\text { W-320- }\end{array}$ & Rev & Status & Discpl & Calculation Title & Originator & Comments & $\begin{array}{l}\text { Issued } \\
\text { Under } \\
\text { HNF. }\end{array}$ \\
\hline 53 & $25-013$ & 1 & App'd & Mech & $\begin{array}{l}\text { Weight and Center of Gravity for Heel } \\
\text { Jet Mock-up }\end{array}$ & RC Campbell & $\begin{array}{l}\text { Equipment Removal Sys: Removal of the Heel } \\
\text { Jet deleted from project. Calc given final } \\
\text { review, retained for record only and not listed } \\
\text { on the drawing index }\end{array}$ & No \\
\hline 54 & 25-014 & 0 & App'd & Mech & Heel Jet Lifting Point Strength Analysis & RC Campbell & $\begin{array}{l}\text { Equipment Removal Sys: Removal of the Heel } \\
\text { Jet deleted from project. Calc given final } \\
\text { review, retained for record only and not listed } \\
\text { on the drawing index }\end{array}$ & No \\
\hline 55 & $25-015$ & 0 & App'd & Mech & Agitator Lifting Point Strength Analysis & RC Campbell & $\begin{array}{l}\text { Equipment Removal Sys: Removal of the } \\
\text { Agitator Pump deleted from project. Calc } \\
\text { given final review, retained for record only } \\
\text { and not listed on the drawing index }\end{array}$ & No \\
\hline 56 & 25-016 & 1 & As-Built & Mech & $\begin{array}{l}\text { Weight and Center of Gravity for } \\
\text { Transfer Pump Mock-up }\end{array}$ & RC Campbell & $\begin{array}{l}\text { Equipment Removal Sys: Removal of the } \\
\text { Trasfer Pump and the use of the Mock-up } \\
\text { deleted from project. Calc checked against } \\
\text { released drawings, retained for record only } \\
\text { and not listed on the drawing index }\end{array}$ & No \\
\hline 57 & $25-017$ & 0 & As-Built & Mech & Flange Thickness of Shielding Plugs & RC Campbell & $\begin{array}{l}\text { Equipment Removal Sys: Shielding plugs used } \\
\text { for installation only and not part of } \\
\text { permanent facility modification. Calc } \\
\text { checked against released drawings, retained } \\
\text { for record only and not listed on the drawing } \\
\text { index }\end{array}$ & No \\
\hline 58 & $25-018$ & 0 & As-Built & Mech & Lifting Capacity of Bails & RC Campbell & $\begin{array}{l}\text { Equipment Removal Sys: Shielding plugs used } \\
\text { for installation only and not part of } \\
\text { permanent facility modification. Calc } \\
\text { checked against released drawings, retained } \\
\text { for record only and not listed ori the drawing } \\
\text { index }\end{array}$ & No \\
\hline 59 & $25 \cdot 019$ & 1 & As-Built & Mech & Pin sizing of Heel Jet Mockup Joints & RC Campbell & $\begin{array}{l}\text { Equipment Removal Sys: Removal of the Heel } \\
\text { Jet deleted from project. Calc checked } \\
\text { against released drawings, retained for record } \\
\text { only and not listed on the drawing index }\end{array}$ & No \\
\hline
\end{tabular}




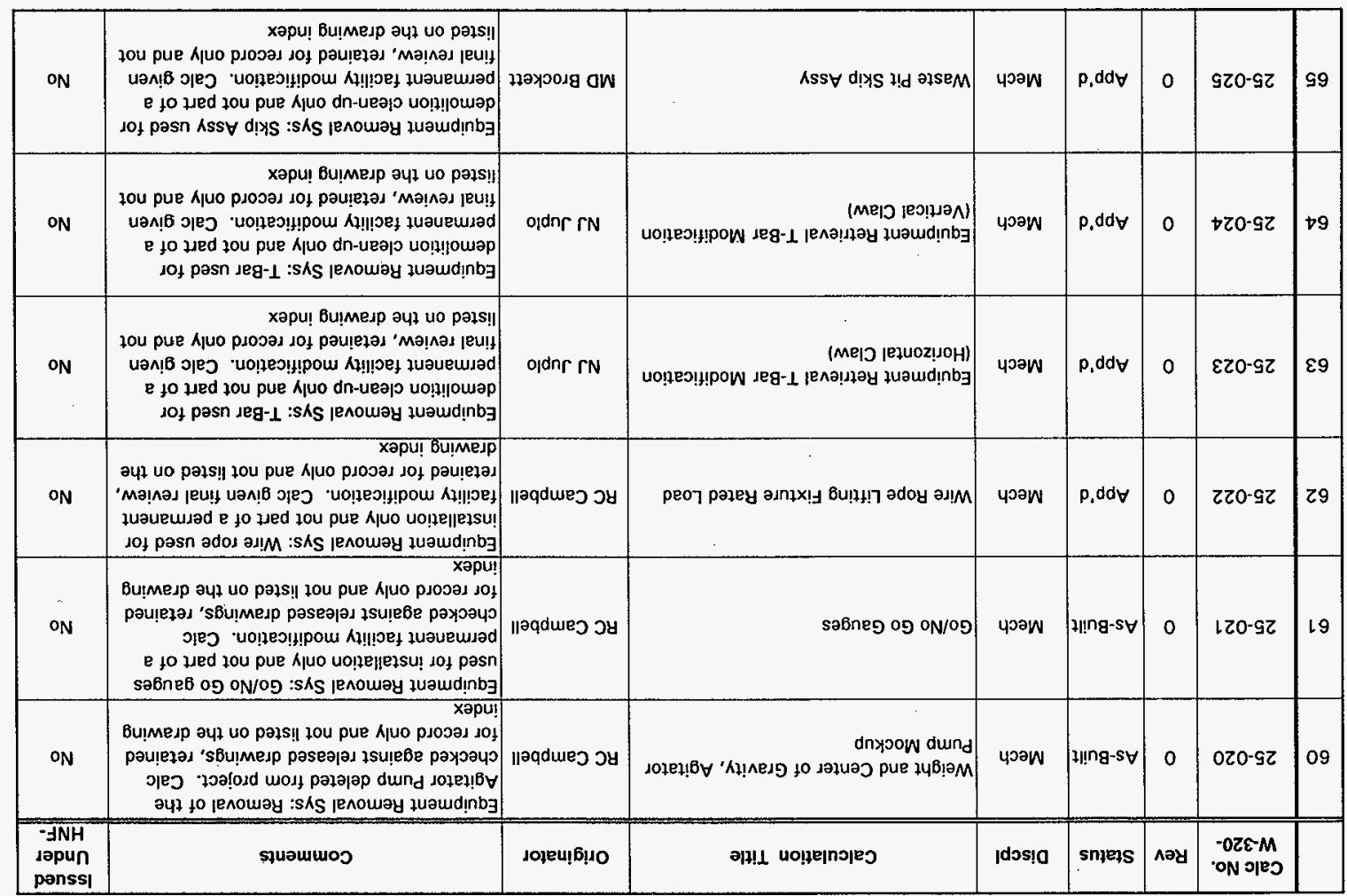


Project W-320 Master Calculation List

\begin{tabular}{|c|c|c|c|c|c|c|c|c|}
\hline & $\begin{array}{l}\text { Calc No. } \\
\text { W-320- }\end{array}$ & Rev & Status & Discpl & Calculation Title & Originator & Comments & $\begin{array}{l}\text { Issued } \\
\text { Under } \\
\text { HNF. } \\
\end{array}$ \\
\hline 66 & $27-001$ & 1 & VOID & Piping & $\begin{array}{l}\text { Sizing of Drain Lines for the Pump Pit } \\
\text { (241-CR-O6A), the Heel Pit (241-CR- } \\
\text { O6B), and the Sluice Pit (241-CR-06C) }\end{array}$ & NJ Juplo & $\begin{array}{l}\text { C-Farm: Heel Pit work deleted. Drain slots } \\
\text { covered and replaced by } 6^{n} \text { dia. drain hole in } \\
\text { sluicer top plate, see } \mathrm{H}-2-818551 \text { sh } 4 \text {. Calc } \\
\text { voided, given final review, retained for record } \\
\text { only and not listed on the drawing index. } \\
\text { Superseded by calc } 27-041\end{array}$ & No \\
\hline 67 & $27-002$ & 1 & VOID & Piping & $\begin{array}{l}\text { Hydraulic Analysis for Slurry Pump } \\
\text { Normal Operations at Maximum } \\
\text { Operational Temperature }\end{array}$ & DL Stone & $\begin{array}{l}\text { C-Farm: Calc voided, given final review, } \\
\text { retained for record only and not listed on the } \\
\text { drawing index. Superseded by calc } 27-048\end{array}$ & No \\
\hline 68 & $27-003$ & 0 & App'd & Piping & Slurry Pumps Hydraulic Calculation & NJ Juplo & $\begin{array}{l}\text { C-Farm: For Pumps P-1361 \& P-1362 and } \\
\text { Line 4" SL-100-M9. Calc not As-Built, given } \\
\text { final review and original content retained for } \\
\text { record, to support Pump P-Spec, W-320-P17, } \\
\text { dated } 1 / 17 / 95 \text {. Superseded by } 27-048\end{array}$ & 2471 \\
\hline 69 & 27-004 & 1 & VOID & Piping & $\begin{array}{l}\text { Hydraulic Analysis for Slurry Pump Start } \\
\text { up Operations at Maximum Operational } \\
\text { Temperature }\end{array}$ & DL Stone & $\begin{array}{l}\text { C-Farm: Calc voided, given final review, } \\
\text { retained for record only and not listed on the } \\
\text { drawing index. Superseded by calc } 27-048\end{array}$ & No \\
\hline 70 & 27.005 & 1 & VOID & Piping & $\begin{array}{l}\text { Hydraulic Analysis for Slurry Pump Start } \\
\text { up Operations at Minimum Operational } \\
\text { Temperature }\end{array}$ & DL Stone & $\begin{array}{l}\text { C-Farm: Calc voided, given final review, } \\
\text { retained for record only and not listed on the } \\
\text { drawing index. Superseded by calc } 27-048\end{array}$ & No \\
\hline 71 & $27-006$ & & $\begin{array}{l}\text { Not } \\
\text { Used }\end{array}$ & & Not Used & & Not Used & NA \\
\hline 72 & $27-007$ & & $\begin{array}{c}\text { Not } \\
\text { Used }\end{array}$ & & Not Used & & Not Used & NA \\
\hline 73 & 27.008 & 1 & VOID & Piping & $\begin{array}{l}\text { Hydraulic Analysis for Sluice Pump at } \\
\text { Maximum Operating Temperature }\end{array}$ & DL. Stone & $\begin{array}{l}\text { AY-Farm: Calc voided, given final review, } \\
\text { retained for record only and not listed on the } \\
\text { drawing index. Superseded by calc } 27-048\end{array}$ & No \\
\hline
\end{tabular}


Project W-320 Master Calculation List

\begin{tabular}{|c|c|c|c|c|c|c|c|c|}
\hline & $\begin{array}{l}\text { Calc No. } \\
W-320-\end{array}$ & Rev & Status & Discpl & Calculation Title & Originator & Comments & $\begin{array}{l}\text { Issued } \\
\text { Under } \\
\text { HNF. }\end{array}$ \\
\hline 74 & $27-009$ & 0 & App'd & Piping & $\begin{array}{l}\text { Hydraulic Analysis for Sluice Pump at } \\
\text { Minimum Operating Temperature }\end{array}$ & K. Cleveland & $\begin{array}{l}\text { AY-Farm: For Pumps P-0621 \& P-0622 and } \\
\text { Line 4" SN-200-M9. Calc not As-Built, given } \\
\text { final review and original content retained for } \\
\text { record, to support Pump P-Spec, W-320-P17, } \\
\text { dated } 1 / 17 / 95 \text {. Superședed by } 27-048\end{array}$ & 2471 \\
\hline 75 & 27-010 & 0 & Void & Piping & $\begin{array}{l}\text { Estimate Water Hammer Due to Sudden } \\
\text { Valve Closure or Sudden Power Loss to } \\
\text { Transfer Pump }\end{array}$ & K Merzouk & $\begin{array}{l}\text { AY, C \& Inter-Farm: Significant pressure } \\
\text { transients due to sudden valve closure only. } \\
\text { Valves on process lines deleted from project. } \\
\text { Calc voided, given final review, retained for } \\
\text { record only and not listed on the drawing } \\
\text { index. }\end{array}$ & No \\
\hline 76 & $27+011$ & 0 & As-Built & Piping & Sizing of Compressor & NJ Juplo & C-Farm: Air Compressor CPR-1361 & 2471 \\
\hline 77 & $27-012$ & 0 & Void & Piping & $\begin{array}{l}\text { Pressure Forces at Changes in Direction } \\
\text { in Transfer Line During Transient States }\end{array}$ & K Merzouk & $\begin{array}{l}\text { AY, C \& Inter-Farm: Significant pressure } \\
\text { transients due to sudden valve closure only. } \\
\text { Valves on process lines deleted from project. } \\
\text { Calc voided, given final review, retained for } \\
\text { record only and not listed on the drawing } \\
\text { index. }\end{array}$ & No \\
\hline 78 & $27-013$ & 3 & As-Built & Piping & Process Pipe Stress Analysis & DL Stone & $\begin{array}{l}\text { AY, C \& Inter-Farm: Lines } 4^{*} \text { SL-100-M9 \& } \\
4^{*} \text { SN-200-M9. Ref calc's 27-016, 029 \& } \\
055\end{array}$ & 2472 \\
\hline 79 & $27-014$ & 3 & As-Built & Piping & Encasement Pipe Stress Analysis & DL Stone & $\begin{array}{l}\text { AY, C \& Inter-Farm: Lines 4" SL-100-M9 \& } \\
\text { 4" SN-200-M9 encasements }\end{array}$ & 2473 \\
\hline 80 & $27-015$ & 3 & As-Built & Piping & Support Structural Analysis & $\begin{array}{l}\text { DL Stone } \\
\text { JR Booth }\end{array}$ & $\begin{array}{l}\text { AY, C \& Inter-Farm: Lines } 4^{\prime \prime} \text { SL-100-M9 \& } \\
\text { 4" SN-200-M9 structural supports }^{\text {S }}\end{array}$ & 2474 \\
\hline 81 & $27-016$ & 2 & As-Built & Piping & C Farm Jumper Stress Analysis & DL Stone & $\begin{array}{l}\text { C-Farm: Pit C-06A (jumper U9-A,B) \& Pit C- } \\
\text { 06C (jumper U6-A). Ref calc's } 27-013 \& \\
055\end{array}$ & 2474 \\
\hline 82 & $27-017$ & 0 & As-Built & Piping & Distributor Stress Analysis & RC Campbell & AY-Farm; Distributor D-0621 & 2474 \\
\hline 83 & 27-018 & 0 & VOID & Piping & Sizing of Pressure Relief Valve & RC Campbell & $\begin{array}{l}\text { C-Farm: Requirement to pressure relieve } \\
\text { process flow }(350 \mathrm{gpm}) \text { deleted from project. } \\
\text { Calc voided, retained for record only and not } \\
\text { listed on the drawing index. }\end{array}$ & No \\
\hline
\end{tabular}


Project W-320 Master Calculation List

\begin{tabular}{|c|c|c|c|c|c|c|c|c|}
\hline & $\begin{array}{l}\text { Calc No. } \\
\text { W-320. }\end{array}$ & Rev & Status & Discpl & Calculation Title & Originator & Comments & $\begin{array}{l}\text { Issued } \\
\text { Under } \\
\text { HNF. }\end{array}$ \\
\hline 84 & $27-019$ & 0 & As-Built & Piping & Winch Analysis & D.L. Stone & AY \& C-Farm: Winches W-0621 \& W-1361 & 2474 \\
\hline 85 & $27-020$ & 0 & App'd & Piping & $\begin{array}{l}\text { Alternate Routing Analysis, } \mathrm{M} 10, \mathrm{M} 11, \\
\mathrm{M} 12 \text {, and } \mathrm{M} 13\end{array}$ & RC Campbell & $\begin{array}{l}\text { AY-Farm: Alternate routing not used, calc } \\
\text { given final review, retained for record only \& } \\
\text { not listed on drawing index }\end{array}$ & No \\
\hline 86 & $27-021$ & 0 & App'd & Piping & Distributor Siphon Breaker & RC Campbell & $\begin{array}{l}\text { AY-Farm: Calc given final review, retained for } \\
\text { record only \& not listed on drawing index. } \\
\text { Superseded by calc's } 27-045 \& 27-048\end{array}$ & No \\
\hline 87 & $27-022$ & 0 & App'd & Piping & Sluice Pump Siphon Break & RC Campbeli & $\begin{array}{l}\text { AY-Farm: Calc given final review, retained for } \\
\text { record only \& not listed on drawing index. } \\
\text { Superseded by calc's } 27-045 \& 27-048\end{array}$ & No \\
\hline 88 & $27-023$ & & $\begin{array}{c}\text { Not } \\
\text { Used }\end{array}$ & & Not Used & & Not Used & NA \\
\hline 89 & $27-024$ & 0 & Void & Piping & Tank Template Drain Flow & $\begin{array}{c}\text { AW } \\
\text { McNichols }\end{array}$ & $\begin{array}{l}\text { AY \& C-Farm: Calc voided, retained for } \\
\text { record only \& not listed on the drawing index. } \\
\text { Superseded by calc } 27-041\end{array}$ & No \\
\hline 90 & $27-025$ & 0 & Void & Piping & Heel Pump System Operating Point & RC Campbell & $\begin{array}{l}\text { C-Farm: Heel Pump deleted from project. } \\
\text { Calc voided, given final review, retained for } \\
\text { record only \& not listed on drawing index. }\end{array}$ & No \\
\hline 91 & $27-026$ & 0 & Void & Piping & Eductor Sizing System Curve & RC Campbell & $\begin{array}{l}\text { C-Farm: Eductor System deleted from } \\
\text { project. Calc voided, given final review, } \\
\text { retained for record only \& not listed on } \\
\text { drawing index. }\end{array}$ & No \\
\hline 92 & $27-027$ & & $\begin{array}{l}\text { Not } \\
\text { Used }\end{array}$ & & Not Used & & Not Used & NA \\
\hline 93 & $27-028$ & 1 & As-Built & Piping & Miscellaneous Pipe Stress Analysis & DL Stone & $\begin{array}{l}\text { C-Farm: Drain Line, 3" DR-302-M9, \& it's 6" } \\
\text { encasement }\end{array}$ & 2475 \\
\hline 94 & $27-029$ & 3 & As-Built & Piping & $\begin{array}{l}\text { Miscellaneous Jumper Pipe Stress. } \\
\text { Analysis - AY Farm }\end{array}$ & DL Stone & $\begin{array}{l}\text { AY-Farm: Pit AY-02E (jumper U2-A,B) \& Pit } \\
\text { AY-02A (jumper U11-A). Ref calc's 27-013 } \\
\text { \& 055 }\end{array}$ & 2475 \\
\hline 95 & $27-030$ & 0 & As-Built & Piping & HVAC Seal Loop & $\begin{array}{c}\text { AW } \\
\text { McNichols }\end{array}$ & $\begin{array}{l}\text { C-Farm: Loops 6" VT-1001-M42 \& 6" VT- } \\
1002-M 42\end{array}$ & 2475 \\
\hline 96 & $27-031$ & 0 & As-Built & Piping & Booster Pump Restrain Analysis & RC Campbell & AY-Farm: Sluice Pump, P-0622 & 2475 \\
\hline
\end{tabular}


Project W-320 Master Calculation List

\begin{tabular}{|c|c|c|c|c|c|c|c|c|}
\hline & $\begin{array}{l}\text { Calc No. } \\
\text { W-320. }\end{array}$ & Rev & Status & Discpl & Calculation Title & Originator & Comments & $\begin{array}{l}\text { Issued } \\
\text { Under } \\
\text { HNF. }\end{array}$ \\
\hline 97 & $27-032$ & 0 & As-Built & Piping & Slurry Pump Seismic Stability & NJ Juplo & C-Farm: Slurry Pump, P-1361 & 2476 \\
\hline 98 & $27-033$ & 0 & Void & Piping & Stress on Sluicer Arm & $\begin{array}{c}\text { AW } \\
\text { McNichols }\end{array}$ & $\begin{array}{l}\text { C-Farm: Calc voided, given final review, } \\
\text { retained for record only \& not listed on the } \\
\text { drawing index. Superseded by calc's } 27-034 \\
\& 27-044\end{array}$ & No \\
\hline 99 & $27-034$ & 0 & As-Built & Piping & Analysis of Sluicer & MB Lasota & C-Farm: Sluicer, S-1361 & 2476 \\
\hline 100 & $27-035$ & 0 & As-Built & Piping & Checking Sluicer Gears for Adequacy & A. Tedrow & C-Farm: Sluicer, S-1361 & 2476 \\
\hline 101 & $27-036$ & 1 & As-Built & Piping & Winch/Pump Rigging Assembly & DL Stone & $\begin{array}{l}\text { AY \& C-Farm: Rigging assembly for winches } \\
\text { W-0621 \& W-1361 }\end{array}$ & 2476 \\
\hline 102 & $27-037$ & 0 & $A p p ' d$ & Piping & $\begin{array}{l}\text { Winch/Pump Simulation Testing } \\
\text { Assembly }\end{array}$ & DL Stone & $\begin{array}{l}\text { AY \& C-Farm; Test assembly used for } \\
\text { training/testing only, not part of a permanent } \\
\text { facility modification. Calc given final review, } \\
\text { retained for record only \& not listed on } \\
\text { drawing index. }\end{array}$ & No \\
\hline 103 & $27-038$ & 0 & App'd & Piping & $\begin{array}{l}\text { Stress Analysis of Encasement Pipe } \\
\text { Buried in Controlled Density Fill }\end{array}$ & MH Ahmed & $\begin{array}{l}\text { AY \& C-Farm: The option to bury encasement } \\
\text { pipe in controlled fill was not used. Calc } \\
\text { given final review, retained for record only \& } \\
\text { not listed on drawing index. }\end{array}$ & No \\
\hline 104 & $27-039$ & 0 & App'd & Piping & Temporary Winch Support Platform & JG Rupert & $\begin{array}{l}\text { AY \& C-Farm: The platform was used to } \\
\text { support installation and was not part of a } \\
\text { permanent facility modification. Calc given } \\
\text { final review, retained for record only \& not } \\
\text { listed on drawing index. }\end{array}$ & No \\
\hline 105 & $27-040$ & 0 & As-Built & Piping & Booster Pump Assembly Components & DL. Stone & $\begin{array}{l}\text { C-Farm: Booster Pump P-1362 seismic } \\
\text { restraint }\end{array}$ & 2476 \\
\hline 106 & $27-041$ & 1 & As-Built & Piping & High Capacity Drain Flow & DL Stone & $\begin{array}{l}\text { AY \& C-Farm: The 6" dia. drain is used in C- } \\
\text { Farm Pits O6A \& 06C and in AY-Farm Pits } \\
\text { O2A \& 02E. Drains have been installed in } \\
\text { Winches W-0621 \& W-1361, Distributor D- } \\
\text { O621, and Sluicer S-1361. Supersedes } \\
\text { calc's 27-001 and 27-024 }\end{array}$ & 2476 \\
\hline 107 & $27-042$ & 0 & As-Built & Piping & Adapter Plate Stress Analysis & DL. Stone & $\begin{array}{l}\text { AY \& C-Farm: Adapter plates for winches W- } \\
1361 \text { \& W-0621 }\end{array}$ & 2476 \\
\hline
\end{tabular}


Project W-320 Master Calculation List

\begin{tabular}{|c|c|c|c|c|c|c|c|c|}
\hline & $\begin{array}{l}\text { Calc No. } \\
\text { W-320- }\end{array}$ & Rev & Status & Discpl & Calculation Title & Originator & Comments & $\begin{array}{l}\text { Issued } \\
\text { Under } \\
\text { HNF- }\end{array}$ \\
\hline 108 & $27-043$ & 0 & As-Built & Piping & Reynolds Numbers at Various \% Solids & DL Stone & $\begin{array}{l}\text { AY, C \& Inter-Farm: Lines } 4^{\prime \prime} \text { SL-100-M9 \& } \\
\text { 4" SN-200-M9 }\end{array}$ & 2476 \\
\hline 109 & $27-044$ & 0 & As-Built & Piping & Sluicer Nozzle Force Analysis & DL Stone & $\begin{array}{l}\text { C-Farm: Hydraulic force of Sluicer (S-1361) } \\
\text { nozzle. Supersedes calc } 27-033\end{array}$ & 2477 \\
\hline 110 & $27-045$ & 0 & As-Built & Piping & Pipe Drain Flows \& Anti-Siphon Sizing & DL Stone & $\begin{array}{l}\text { AY-Farm: Lines 4" SL-100-M9 (D-0621) \& 4" } \\
\text { SN-200-M9 (W-0621) }\end{array}$ & 2477 \\
\hline 111 & $27-046$ & 0 & As-built & Piping & Flushing Nozzle Lifting Force & TK Peterson & $\begin{array}{l}\text { AY-Farm: Lifting force on shielding plugs in } \\
\text { cover blocks for AY-Farm's pits O2A \& O2E }\end{array}$ & 2477 \\
\hline 112 & $27-047$ & 0 & As-Built & Piping & Pit Volume and In/Out Flow Areas & MR Custer & $\begin{array}{l}\text { AY \& C-Farm: 6C's Pits 06A \& } 06 C \text { and } \\
\text { 2AY's Pits 02A \& O2E }\end{array}$ & 2477 \\
\hline 113 & $27-048$ & 0 & As-Built & Piping & Slurry/Supernate Hydraulic Analysis & K Hayase & $\begin{array}{l}\text { AY, C \& Inter-Farm: Lines 4" SL-100-M9 \& } \\
4^{\prime \prime} \text { SN-200-M9 and their appurtenances. As- } \\
\text { Built Slurry ard Sluice Systems Hydraulic } \\
\text { Calc. Supersedes Calc's 27-002, 003, 004, } \\
005,008,009,021 \& 022 \text {. }\end{array}$ & 2478 \\
\hline 114 & $27-049$ & 0 & As-Built & Piping & Size of HVAC Duct Flushing System & NJ Juplo & $\begin{array}{l}\text { C-Farm \& HVAC Skid: Duct, 10" VT-1010- } \\
\text { M8 }\end{array}$ & 2479 \\
\hline 115 & $27-050$ & 0 & App'd & Piping & Portable Exhauster Installation Analysis & K Hayase & $\begin{array}{l}\text { AY \& C-Farm: Portable exhausters are for } \\
\text { emergency back-up only and are not } \\
\text { installed. Calc given final review but not As- } \\
\text { Build. }\end{array}$ & 2479 \\
\hline 116 & $27-051$ & 0 & As-Built & Piping & Jumper Flushing Supply Head Analysis & K Hayase & $\begin{array}{l}\text { AY-Farm: Pits O2A \&02E jumpers to support } \\
\text { flushing lines } 4^{*} \text { SL-100-M9 \& } 4^{*} \text { SN-200- } \\
\text { M9 }\end{array}$ & 2479 \\
\hline 117 & $27-052$ & 0 & App'd & Piping & $\begin{array}{l}\text { AY-102 Annulus Supply Air Filter } \\
\text { Housing Installation Analysis }\end{array}$ & K Hayase & $\begin{array}{l}\text { AY-Farm: Filter housing used for testing only } \\
\text { and removed before project turn over. Calc } \\
\text { given final review, retained for record only \& } \\
\text { not listed on the drawing index. }\end{array}$ & 2479 \\
\hline 118 & $27-053$ & 0 & As-Built & Piping & New Supernate Jumper Analysis & K Hayase & AY-Farm: Pit 02A, (jumper U3-U11-B) & 2479 \\
\hline 119 & $27-054$ & 0 & App'd & Piping & $\begin{array}{l}\text { System Curves for Portable Exhauster } \\
\text { Installation }\end{array}$ & K Hayase & $\begin{array}{l}\text { AY \& C-Farm: System curves for portable } \\
\text { exhausters to be used for } 6 \text { C's dome exhaust } \\
\text { air and } 2 \text { AY's dome \& annulus exhaust air. } \\
\text { Portable exhausters are not installed. Calc } \\
\text { given final review but not As-Built. }\end{array}$ & 2479 \\
\hline
\end{tabular}

Page 11 of 17 
Project W-320 Master Calculation List

\begin{tabular}{|c|c|c|c|c|c|c|c|c|}
\hline & $\begin{array}{l}\text { Calc No. } \\
\text { W-320. }\end{array}$ & Rev & Status & Discpl & Calculation Title & Originator & Comments & $\begin{array}{l}\text { Issued } \\
\text { Under } \\
\text { HNF. }\end{array}$ \\
\hline 120 & $27-055$ & 0 & As-Built & Piping & $\begin{array}{l}\text { Nozzle Plate \& Encasement Plate } \\
\text { Analysis }\end{array}$ & M Ahmed & $\begin{array}{l}\text { AY \& C-Farm: Wail nozzle analysis for } \\
\text { jumpers U9-A,B (pit C-06A), U6-A (pit C- } \\
\text { O6C), U2-A,B (pit AY-02E) and U11-A (pit AY } \\
\text { O2A). Ref calc's 27-013,016 \& } 029 \text {. }\end{array}$ & 2479 \\
\hline 121 & $28-001$ & 1 & As-built & HVAC & $\begin{array}{l}\text { Exhaust Airflow Sizing for Tank 241-C- } \\
106\end{array}$ & PH Langowski & $\begin{array}{l}\text { C-Farm: Exhaust system airflow component } \\
\text { sizing to include; HEME, HEMF, exhaust skid, } \\
\text { and exhaust Fan FN- } 1362 \text {. Ref calc's } 28- \\
004 \& 008\end{array}$ & 2483 \\
\hline 122 & $28-002$ & 0 & Void & HVAC & Directed Air System & N/A & $\begin{array}{l}\text { C-Farm: Directed Air System deleted from } \\
\text { project. Calc voided, given final review, } \\
\text { retained for record only and not listed on the } \\
\text { drawing index. Renumbered from } \mathrm{H}-0 \mathrm{O} 2 \text { to. } \\
28-002 \text {. }\end{array}$ & No \\
\hline 123 & $28-003$ & 0 & As-built & HVAC & $\begin{array}{l}\text { Equipment Sizing \& Selection } \\
\text { Recirculation Fan }\end{array}$ & PH Langowski & $\begin{array}{l}\text { HVAC Skid: Recirculation Fan FN-1362. } \\
\text { Renumbered from } \mathrm{H}-003 \text { to } 28-003\end{array}$ & 2483 \\
\hline 124 & $28-004$ & 1 & As-built & HVAC & Sizing High Efficiency Mist Eliminator & RA Pina & $\begin{array}{l}\text { HVAC Skid: Mist Eliminator HME-1361 } \\
\text { Renumbered from } \mathrm{H}-004 \text { to } 28-004\end{array}$ & 2483 \\
\hline 125 & $28-005$ & 1 & As-built & HVAC & Sizing Electric Heating Coil & RA Pina & $\begin{array}{l}\text { HVAC Skid: Recirculation Heater HC-1361 } \\
\text { Renumbered from } \mathrm{H}-005 \text { to } 28-005\end{array}$ & 2483 \\
\hline 126 & $28-006$ & 0 & As-built & HVAC & $\begin{array}{l}\text { Equipment Sizing \& Selection, } \\
\text { Recirculation Condenser }\end{array}$ & CT O'Neill & $\begin{array}{l}\text { HVAC Skid: Recirc Condenser } \mathrm{HX}-1361 \\
\text { Renumbered from } \mathrm{H}-006 \text { to } 28-006\end{array}$ & 2483 \\
\hline 127 & $28-007$ & 0 & As-built & HVAC & Chiller Skid System Sizing and Selection & R Abjelina & $\begin{array}{l}\text { C-Farm: Sizing Chiller Skid Components. } \\
\text { Calc done by offsite subcontractor }\end{array}$ & 2483 \\
\hline 128 & $28-008$ & 1 & As-built & HVAC & $\begin{array}{l}\text { High Efficiency Metal Filter Shielding } \\
\text { Input and Flushing Frequency }\end{array}$ & PH Langowski & $\begin{array}{l}\text { HVAC Skid: Metal Filter HMF-1361 } \\
\text { Renumbered from H-008 to } 28-008\end{array}$ & 2483 \\
\hline 129 & 28-009 & & $\begin{array}{l}\text { Not } \\
\text { Used }\end{array}$ & & Not Used & & Not Used & NA \\
\hline 130 & 28-010 & & $\begin{array}{l}\text { Not } \\
\text { Used }\end{array}$ & & Not Used & & Not Used & NA \\
\hline 131 & 28-011 & 1 & As-built & HVAC & $\begin{array}{l}\text { Exhaust Skid Stack Sizing and Fan } \\
\text { Sizing }\end{array}$ & PH Langowski & $\begin{array}{l}\text { C-Farm: Stack } 296-\mathrm{C}-006 \text {, Fan FN-1362, and } \\
\text { Heater HC-1362. Renumbered from } \mathrm{H}-011 \\
\text { to } 28-011\end{array}$ & 2483 \\
\hline
\end{tabular}


Project W-320 Master Calculation List

\begin{tabular}{|c|c|c|c|c|c|c|c|c|}
\hline & $\begin{array}{l}\text { Calc No. } \\
\text { W-320- }\end{array}$ & Rev & Status & Discpl & Calculation Title & Originator & Comments & $\begin{array}{l}\text { Issued } \\
\text { Under } \\
\text { HNF. }\end{array}$ \\
\hline 132 & $28-012$ & 0 & As-built & HVAC & Process Building HVAC & RA Pina & $\begin{array}{l}\text { C-Farm: Process bldg's Fan FN-1364, Filter F- } \\
1368 \text { and Heaters UH-1362 \& UH-1361. } \\
\text { Renumbered from H-012 to } 28-012\end{array}$ & 2484 \\
\hline 133 & $28-013$ & 0 & As-built & HVAC & $\begin{array}{l}\text { Chilled Water Line } \\
\text { Expansion/Contraction and Heat Gain }\end{array}$ & R Abjelina & $\begin{array}{l}\text { C-Farm: Lines } 3^{n} \text { CWS-803-M4 \& } 3^{n} \text { CWR- } \\
850-M 4 \text {. Calc done by offsite subcontractor. } \\
\text { Renumbered from H-013 to } 28-013\end{array}$ & 2484 \\
\hline 134 & $28-014$ & 0 & App'd & HVAC & $\begin{array}{l}\text { Air Flow Calc System Review for } \\
\text { Capture Velocity }\end{array}$ & RG Hale & $\begin{array}{l}\text { Equipment Removal Sys: Used to support } \\
\text { equipment removal and not part of permanent } \\
\text { facility modification. Calc given final review, } \\
\text { retained for record only and not listed on } \\
\text { drawing index. }\end{array}$ & No \\
\hline 135 & $28-015$ & 1 & As-built & HVAC & Sizing of Moisture Separator & RA Pina & $\begin{array}{l}\text { HVAC Skid: Moisture Separator MS-1361 } \\
\text { Renumbered from H-015 to } 28-015\end{array}$ & 2484 \\
\hline 136 & $28-016$ & 0 & As-built & HVAC & Pressure Relief Seal Loop Sizing & PH Langowski & $\begin{array}{l}\text { C-Farm: 6" VT-1001-M42 \& 6" VT-1002-M9, } \\
\text { Ref calc's 28-020 (commercial grade } \\
\text { analysis) and 28-031 (independent } \\
\text { verification). Renumbered from H-016 to 28- } \\
016\end{array}$ & 2484 \\
\hline 137 & $28-017$ & 2 & As-built & HVAC & $\begin{array}{l}\text { Service Building Fan Selection and } \\
\text { Heating Load }\end{array}$ & RA Pina & $\begin{array}{l}\text { Inter-Farm: Air \& Water Service Bldg's Filter } \\
\text { F-1366, Fan FN-1363, and Heater HC-1363 } \\
\text { Renumbered from H-017 to } 28-017\end{array}$ & 2484 \\
\hline 138 & $28-018$ & 1 & As-built & HVAC & $\begin{array}{l}\text { Pressure Loss Upstream of the Exhaust } \\
\text { Skid }\end{array}$ & PH Langowski & $\begin{array}{l}\text { HVAC Skid \& C-Farm: Fans FN-1361 and FN- } \\
\text { 1362. Renumbered from } \mathrm{H}-018 \text { to } 28-018\end{array}$ & 2484 \\
\hline 139 & 28-019 & & $\begin{array}{l}\text { Not } \\
\text { Used }\end{array}$ & & Not Used & & Not Used & NA \\
\hline 140 & 28.020 & 0 & As-built & HVAC & $\begin{array}{l}\text { Commercial Grade Item Analysis, } \\
\text { Pressure Relief Seal Loop }\end{array}$ & PH Langowski & $\begin{array}{l}\text { C-Farm: } 6^{\prime \prime} \text { VT-1001-M42 \& } 6^{*} \mathrm{VT}-1002- \\
\text { M42. Ref calc's } 28-020 \text { (seal loop sizing) \& } \\
\text { 28-031 (independent verification). } \\
\text { Renumbered from H-020 to 28-020 }\end{array}$ & 2484 \\
\hline 141 & $28-021$ & 0 & As-built & HVAC & $\begin{array}{l}\text { Above Ground Pipe Stress Analysis for } \\
\text { Duct Piping }\end{array}$ & DL Stone & $\begin{array}{l}\text { C-Farm: } 10^{n} \mathrm{VT}-1010-\mathrm{M} 8,8^{n} \mathrm{VT}-1022-\mathrm{M} 8 \text {, } \\
\text { and } 6^{\prime \prime} \mathrm{VT}-1060-\mathrm{M} 8 \text { Renumbered from } \mathrm{H}- \\
021 \text { to } 28-021\end{array}$ & 2485 \\
\hline
\end{tabular}


Project W-320 Master Calculation List

\begin{tabular}{|c|c|c|c|c|c|c|c|c|}
\hline & $\begin{array}{l}\text { Calc No. } \\
\text { W-320- }\end{array}$ & Rev & Status & Discpl & Calculation Title & Originator & Comments & $\begin{array}{l}\text { Issued } \\
\text { Under } \\
\text { HNF. }\end{array}$ \\
\hline 142 & $28-022$ & 0 & As-built & HVAC & Seal Pot Sizing for Leak Detection & RA Pina & $\begin{array}{l}\text { C-Farm: Process bldg } 241-\mathrm{C}-91 \text { 's seal pot } \\
\text { sizing. Renumbered from } \mathrm{H}-022 \text { to } 28-022\end{array}$ & 2485 \\
\hline 143 & $28-023$ & 1 & As-built & HVAC & $\begin{array}{l}\text { Cooling and Heating Required for } \\
\text { Seismic Switch Missile Enclosure }\end{array}$ & PH Langowski & $\begin{array}{l}\text { AY \& C-Farm: Heating/Cooling Units; AC- } \\
1361, \mathrm{AC}-1362, \mathrm{AC}-0621 \text {, and AC-0622. } \\
\text { Renumbered from } \mathrm{H}-023 \text { to } 28-023\end{array}$ & 2485 \\
\hline 144 & $28-024$ & 2 & As-built & HVAC & $\begin{array}{l}\text { Cooling and Heating Required for } \\
\text { Variable Speed Drive (VSD) Enclosure }\end{array}$ & PH Langowski & $\begin{array}{l}\text { AY-Farm: Enclosure Skid } 241-A Y-51 . \\
\text { Renumbered from H-024 to } 28-024\end{array}$ & 2485 \\
\hline 145 & $28-025$ & 1 & As-built & HVAC & $\begin{array}{l}\text { Cooling and Heating Required for Motor } \\
\text { Control Center (MCC) Enclosure }\end{array}$ & PH Langowski & $\begin{array}{l}\text { C-Farm: Enclosure Skid } 241-\mathrm{C}-51 \text {. } \\
\text { Renumbered from } \mathrm{H}-025 \text { to } 28-025\end{array}$ & 2485 \\
\hline 146 & $28-026$ & 0 & As-built & HVAC & Sizing of Ventilation Insulation & RA Pina & $\begin{array}{l}\text { HVAC Skid: Process Bldg 241-C-91 } \\
\text { insulation. Renumbered from } \mathrm{H}-026 \text { to } 28 \text { - } \\
026\end{array}$ & 2485 \\
\hline 147 & $28-027$ & 1 & As-built & HVAC & $\begin{array}{l}\text { Above Ground Pipe Support Stress } \\
\text { Analysis }\end{array}$ & RC Campbell & $\begin{array}{l}\text { HVAC Skid: Bldg } 241-\mathrm{C}-91 \text { duct supports } \\
\text { Renumbered from } \mathrm{H}-027 \text { to } 28-027\end{array}$ & 2485 \\
\hline 148 & $28-028$ & N/A & Void & N/A & N/A & N/A & & NA \\
\hline 149 & $28-029$ & 1 & As-built & HVAC & Cooling Load in Pump Pit 241-C-06A & RA Pina & $\begin{array}{l}\text { C-Farm: Pump Pit O6A cooling load. } \\
\text { Renumbered from } \mathrm{H}-029 \text { to } 28-029\end{array}$ & 2485 \\
\hline 150 & $28-030$ & 1 & As-built & HVAC & Cooling Load in Pump Pit 241-AY-102 & WJ Hodges & $\begin{array}{l}\text { AY-Farm: Sluice Pit AY-02E cooling load. } \\
\text { Chiller R-0621 \& Fan/Coil FC-0621. } \\
\text { Renumbered from H-030 to 28-030 }\end{array}$ & 2486 \\
\hline 151 & $28-031$ & 0 & As-built & HVAC & $\begin{array}{l}\text { Pressure Relief Seal Loop Design } \\
\text { Verification }\end{array}$ & EL Dalpiaz & $\begin{array}{l}\text { C-Farm: 6" VT-1001-M42 \& 6" VT-1002- } \\
\text { M42. Ref calc's } 28-016 \text { (seal loop sizing) \& } \\
\text { 28-020 (commercial grade analysis). } \\
\text { Renumbered from H-031 to 28-031 }\end{array}$ & 2486 \\
\hline 152 & $28-032$ & 0 & As-built & HVAC & Process Building Piping Stress Analysis & RC Campbelf & $\begin{array}{l}\text { HVAC Skid: Process Bldg 241-C-91 dome } \\
\text { exhaust air piping (ducting). Renumbered } \\
\text { from } \mathrm{H}-032 \text { to } 28-032\end{array}$ & 2486 \\
\hline 153 & $28-033$ & 0 & As-built & HVAC & $\begin{array}{l}\text { Process Building Duct Support Stress } \\
\text { Analysis }\end{array}$ & RC Campbell & $\begin{array}{l}\text { HVAC Skid: Process Bldg 241-C-91 pipe } \\
\text { (duct) supports. Renumbered from } \mathrm{H}-033 \\
\text { to } 28-033\end{array}$ & 2486 \\
\hline
\end{tabular}


Project W-320 Master Calculation List

\begin{tabular}{|c|c|c|c|c|c|c|c|c|}
\hline & $\begin{array}{l}\text { Calc No. } \\
\text { W-320- }\end{array}$ & Rev & Status & Discpl & Calculation Title & Originator & Comments & $\begin{array}{l}\text { Issued } \\
\text { Under } \\
\text { HNF. }\end{array}$ \\
\hline 154 & $28-034$ & 0 & As-built & HVAC & $\begin{array}{l}\text { Exhaust Skid Maximum Allowable } \\
\text { Leakage Criteria }\end{array}$ & PH Langowski & $\begin{array}{l}\text { C-Farm: Exhaust Skid pressure decay leak } \\
\text { test requirements. Renumbered from } \mathrm{H}-034 \\
\text { to } 28-034\end{array}$ & 2486 \\
\hline 155 & $28-035$ & 0 & As-built & HVAC & $\begin{array}{l}\text { Recirculation Heater, N509 Duct } \\
\text { Requirements }\end{array}$ & RA Pina & $\begin{array}{l}\text { HVAC Skid: Process Bldg recirculation heater } \\
\mathrm{HC}-1361 \text {. Renumbered from } \mathrm{H}-035 \text { to } 28- \\
035\end{array}$ & 2486 \\
\hline 156 & $31-001$ & 0 & As-built & Elect & Fault Current Analysis & $\begin{array}{l}\text { JC Del } \\
\text { Rosario }\end{array}$ & Equipment Removal Sys: & 2487 \\
\hline 157 & $31-002$ & 0 & As-built & Elect & Standby Generator Sizing & $\begin{array}{l}\text { JC Del } \\
\text { Rosario }\end{array}$ & Equipment Removal Sys: & 2487 \\
\hline 158 & $31-003$ & 0 & As-built & Elect & Feeder Cable Sizes \& \% Voltage Drop & $\begin{array}{l}\text { JC Del. } \\
\text { Rosario }\end{array}$ & Equipment Removal Sys: & 2487 \\
\hline 159 & $31-004$ & 0 & App'd & Elect & Transformer Sizing & $\begin{array}{c}\text { DL } \\
\text { Levandowski }\end{array}$ & $\begin{array}{l}\text { Equipment Removal Sys: Done to Support } \\
\text { Construction Trailer Installation. }\end{array}$ & No \\
\hline 160 & 31-005 & 0 & App'd & Elect & $\begin{array}{l}\text { Total Loads for Lunchroom Trailer \& } \\
\text { Office Trailer }\end{array}$ & $\begin{array}{c}\text { DL. } \\
\text { Levandowski }\end{array}$ & $\begin{array}{l}\text { Equipment Removal Sys: Done to Support } \\
\text { Construction Trailer Installation. }\end{array}$ & No \\
\hline 161 & $31-006$ & 0 & App'd & Elect & Total Loads for Fabrication shop & $\begin{array}{c}\mathrm{DL} \\
\text { Levandowski }\end{array}$ & $\begin{array}{l}\text { Equipment Removal Sys: Done to Support } \\
\text { Construction Trailer Installation. }\end{array}$ & No \\
\hline 162 & $31-007$ & 1 & App'd & Elect & $\begin{array}{l}\text { Total Loads for Fabrication Shop, } \\
\text { Lunchroom Trailer \& Office Trailer }\end{array}$ & $\frac{\mathrm{DL}}{\text { Levandowski }}$ & $\begin{array}{l}\text { Equipment Remova! Sys: Done to Support } \\
\text { Construction Trailer Installation. }\end{array}$ & No \\
\hline 163 & $31-008$ & 1 & App'd & Elect & Wireway Sizes for Rack Details & $\begin{array}{c}\text { DL. } \\
\text { Levandowski }\end{array}$ & $\begin{array}{l}\text { Equipment Removal Sys: Done to Support } \\
\text { Construction Trailer Installation. }\end{array}$ & No \\
\hline 164 & 31.009 & 1 & & Elect & $\begin{array}{l}\text { Conduit, Nipple, conductor, Grounding } \\
\text { \& Trip Setting sizing }\end{array}$ & $\begin{array}{c}\mathrm{DL} \\
\text { Levandowski }\end{array}$ & $\begin{array}{l}\text { Equipment Removal Sys: Done to Support } \\
\text { Construction Trailer Installation. }\end{array}$ & No \\
\hline 165 & $31-010$ & 1 & As-built & Elect & $\begin{array}{l}\text { C-106 Demand Load Analysis, Size } \\
\text { Feeders, Voltage Drop, and Fault Study }\end{array}$ & TJ Kasnick & C-Farm: & 2487 \\
\hline 166 & $31-011$ & 1 & As-built & Elect & $\begin{array}{l}\text { Tank C-106 Load Determination, } \\
\text { Feeder/Protection Sizing, Voltage Drop, } \\
\text { Lighting Calculations \& Conduit Sizing. }\end{array}$ & DE L.arson & C-Farm: & 2487 \\
\hline 167 & $31-012$ & & $\begin{array}{c}\text { Not } \\
\text { Used }\end{array}$ & & Not Used & & Not Used & NA \\
\hline
\end{tabular}


Project W-320 Master Calculation List

\begin{tabular}{|c|c|c|c|c|c|c|c|c|}
\hline & $\begin{array}{l}\text { Calc No. } \\
\text { W-320- }\end{array}$ & Rev & Status & Discpl & Calculation Title & Originator & Comments & $\begin{array}{l}\text { Issued } \\
\text { Under } \\
\text { HNF- }\end{array}$ \\
\hline 168 & $31-013$ & 0 & App'd & Elect & Cable Pulling Tension -C-Farm & DE Larson & $\begin{array}{l}\text { C-Farm: Not required for design will be used } \\
\text { to direct wire installation during construction. }\end{array}$ & No \\
\hline 169 & $31-014$ & 0 & As-built & Elect & C-Farm Heat Tracing & RH Graeber & C-Farm: & 2488 \\
\hline 170 & $31-015$ & 2 & As-built & Elect & $\begin{array}{l}\text { Service Building Electrical Equipment } \\
\text { Sizing. }\end{array}$ & DE Larson & Site Work: & 2488 \\
\hline 171 & $.31-016$ & 1 & As-built & Elect & $\begin{array}{l}\text { AY-102 Demand Load Analysis, Size } \\
\text { Feeders, Voltage Drop and Fault Study }\end{array}$ & TJ Kasnick & AY-Farm: & 2487 \\
\hline 172 & $31-017$ & 1 & As-built & Elect & $\begin{array}{l}\text { Tank AY-102 Load Determination, } \\
\text { Feeder/Protection Sizing, Voltage Drop } \\
\text { and Lighting Calculations }\end{array}$ & RH Graeber & AY-Farm: & 2487 \\
\hline 173 & $31-018$ & & $\begin{array}{c}\text { Not } \\
\text { Used }\end{array}$ & Elect & Not Used & & Not Used & NA \\
\hline 174 & $31-019$ & 0 & Prelm & Elect & Cable Pulling Tension - AY-Farm & DE Larson & $\begin{array}{l}\text { AY-Farm: Not required for design will be used } \\
\text { to direct wire installation during construction. }\end{array}$ & No \\
\hline 175 & $31-020$ & & $\begin{array}{l}\text { Not } \\
\text { Used }\end{array}$ & Elect & Not Used & & Not Used & NA \\
\hline 176 & $31-021$ & 0 & N/A & Elect & $\begin{array}{l}\text { Load Calculations for Control/Lunch } \\
\text { Room Trailer }\end{array}$ & F Morales & $\begin{array}{l}\text { Control/Lunch Room: Package Voided By } \\
\text { Descoping }\end{array}$ & No \\
\hline 177 & 31.022 & 1 & As-built & Elect & $\begin{array}{l}\text { Control Circuit Sizing \& Voltage Drop } \\
\text { Analysis for Seismic Shutdown System } \\
\text { (Class 1E) }\end{array}$ & $\stackrel{\text { M }}{\text { Khorsandian }}$ & AY \& C-Farm: & 2488 \\
\hline 178 & $31-023$ & 0 & Void & Elect & $\begin{array}{l}\text { Control System to AY-farm Wire Sizing, } \\
\text { Voltage Drop and Conduit Sizing }\end{array}$ & SA Najjar & Control Room: Cancelled by Descoping & No \\
\hline 179 & $31-024$ & 0 & Void & Elect & $\begin{array}{l}\text { Control System to C-Farm Wire Sizing, } \\
\text { Voltage Drop and Conduit Sizing }\end{array}$ & SA Najjar & Control Room: Cancelled by Descoping & No \\
\hline 180 & 33-001 & 0 & App'd & Mech & $\begin{array}{l}\text { Exposure Rate with Pump Vertically } \\
\text { Above Open Pit (Tank C-106) Pump Pit }\end{array}$ & G.J Peter & $\begin{array}{l}\text { C-Farm: This calc was used to determined } \\
\text { radiation fields during demolition and was not } \\
\text { part of a permanent facility modification. } \\
\text { Calc given final review, retained for record } \\
\text { only \& not listed on drawing index. }\end{array}$ & No \\
\hline
\end{tabular}


Project W-320 Master Calculation List

\begin{tabular}{|c|c|c|c|c|c|c|c|c|}
\hline & $\begin{array}{l}\text { Calc No. } \\
\text { W-320- }\end{array}$ & $\operatorname{Rev}$ & Status & Discpl & Calculation Title & Originator & Comments & $\begin{array}{l}\text { Issued } \\
\text { Under } \\
\text { HNF. }\end{array}$ \\
\hline 181 & $33-002$ & 0 & $A p p^{+} d$ & Mech & $\begin{array}{l}\text { Plug Thickness at End of Riser } 42^{\prime \prime} \text { Riser } \\
\text { (ID = 32") Pit CR-06A Tank C-106 }\end{array}$ & GJ Peter & $\begin{array}{l}\text { C-Farm: This calc was used to size a } \\
\text { shielding plug to be used during demolition } \\
\text { and was not part of a permanent facility } \\
\text { modification. Calc given final review, } \\
\text { retained for record only \& not listed on } \\
\text { drawing index. }\end{array}$ & No \\
\hline 182 & $33-003$ & 0 & App'd & Mech & $\begin{array}{l}\text { Above Pit Shielding Thickness with } \\
\text { Pump or Plug in Place - Pit CR - 06A } \\
\text { (Tank C-106) }\end{array}$ & GJ Peter & $\begin{array}{l}\text { C-Farm: This calc was used to size a } \\
\text { shielding plug to be used during demolition } \\
\text { and was not part of a permanent facility } \\
\text { modification. Calc given final review, } \\
\text { retained for record only \& not listed on } \\
\text { drawing index. }\end{array}$ & No \\
\hline 183 & $33-004$ & 1 & As-Built & Mech & $\begin{array}{l}\text { Earth Cover Shielding Thickness for } \\
\text { Project } W-320\end{array}$ & SR Gedeon & $\begin{array}{l}\text { AY, C \& Inter-Farm: Lines } 4^{n} \text { SL-100-M9 \& } \\
\text { 4" SN-200-M9 earth cover shielding. Calc } \\
\text { done by onsite (WHC) subcontractor. }\end{array}$ & 2489 \\
\hline 184 & $33-005$ & 1 & As-Built & Mech & $\begin{array}{l}\text { Cover Block, Sidewall Shielding } \\
\text { Thickness for Pump Pit 241-C-06A and } \\
\text { Stuice Pit 241-C-06C }\end{array}$ & CT Li & C-Farm: Pit 241-C-06A shielding & 2489 \\
\hline 185 & $33-006$ & 0 & As-Built & Mech & $\begin{array}{l}\text { Individual Shielding Thickness for HEMF } \\
\text { or HEME }\end{array}$ & GJ Peter & $\begin{array}{l}\text { HVAC Skid: HME-1361 \& HMF-1361 } \\
\text { shielding }\end{array}$ & 2489 \\
\hline 186 & $33-007$ & 1 & As-Built & Mech & $\begin{array}{l}\text { Shielding Thickness to Reduce } 10 \\
\mathrm{mR} / \mathrm{Hr} \text { to } 2 \mathrm{mR} / \mathrm{Hr} \text { Using (A) Solid Steel } \\
\text { Plate or (B) Air Gap }\end{array}$ & CT Li & HVAC Skid: Bldg 241-C-91 shielding wall & 2489 \\
\hline 187 & $33-008$ & 1 & As-Built & Mech & $\begin{array}{l}\text { Determine if Shielding Necessary for } \\
10^{*} \text { Vapor Pipe from Tank Dome to } \\
\text { HVAC System }\end{array}$ & $\mathrm{CT} \mathrm{Li}$ & C-Farm: Line 10" VT-1010-M8 shielding & 2489 \\
\hline 188 & 33-009 & 0 & As-Built & Mech & $\begin{array}{l}\text { Shielding Calculation for Pump Pit AY-- } \\
02\end{array}$ & SR Gedeon & $\begin{array}{l}\text { AY-Farm: Pits 241-AY-02A \& 241-AY-O2E } \\
\text { shielding. Calc done by onsite (WHC) } \\
\text { subcontractor }\end{array}$ & 2489 \\
\hline 189 & $33-010$ & 0 & App'd & Mech & $\begin{array}{l}\text { Determine the Worst Exposure Rate } \\
\text { Inside the Pump Pit } 241-\mathrm{C}-06 \mathrm{~A}\end{array}$ & GJ Peter & $\begin{array}{l}\text { C-Farm: This calc was used to estimate } \\
\text { exposure rates for an in Pit }(\mathrm{C}-06 \mathrm{~A}) \text { entry } \\
\text { during demolition and was not part of a } \\
\text { permanent facility modification. Calc given } \\
\text { final review, retained for record only \& not } \\
\text { listed on drawing index. }\end{array}$ & No \\
\hline 190 & $34-001$ & 0 & As-Built & Cthdc & Rectifier 46 (Cross-Site Transfer Lines) & AR Snowhite & Site work: & 2492 \\
\hline
\end{tabular}




\section{DISTRIBUTION SHEET}

\begin{tabular}{|c|c|c|c|c|c|}
\hline \multirow{2}{*}{$\begin{array}{l}\text { To } \\
\text { Distribution }\end{array}$} & \multirow{2}{*}{\multicolumn{3}{|c|}{$\begin{array}{l}\text { From } \\
\text { JW Bailey }\end{array}$}} & \multicolumn{2}{|c|}{ Page 1 of 1} \\
\hline & & & & \multicolumn{2}{|c|}{ Date 6 August 1998} \\
\hline \multicolumn{4}{|l|}{ Project Title/Work Order } & \multicolumn{2}{|c|}{ EDT No. 624730} \\
\hline Project $W-320$, HNF-2527 & Calculation Li & & & \multicolumn{2}{|c|}{ ECN No. $n / a$} \\
\hline Name & MSIN & $\begin{array}{l}\text { Text } \\
\text { With All } \\
\text { Attach. }\end{array}$ & Text Only & $\begin{array}{l}\text { Attach./ } \\
\text { Appendix } \\
\text { Only }\end{array}$ & $\begin{array}{c}\text { EDT/ECN } \\
\text { Only }\end{array}$ \\
\hline $\begin{array}{l}\text { JW Bailey } \\
\text { DE Evans } \\
\text { W-320 Project Files }\end{array}$ & $\begin{array}{l}S 2-48 \\
S 2-47 \\
\mathrm{RI}-29 \\
\end{array}$ & & & & \\
\hline
\end{tabular}

